
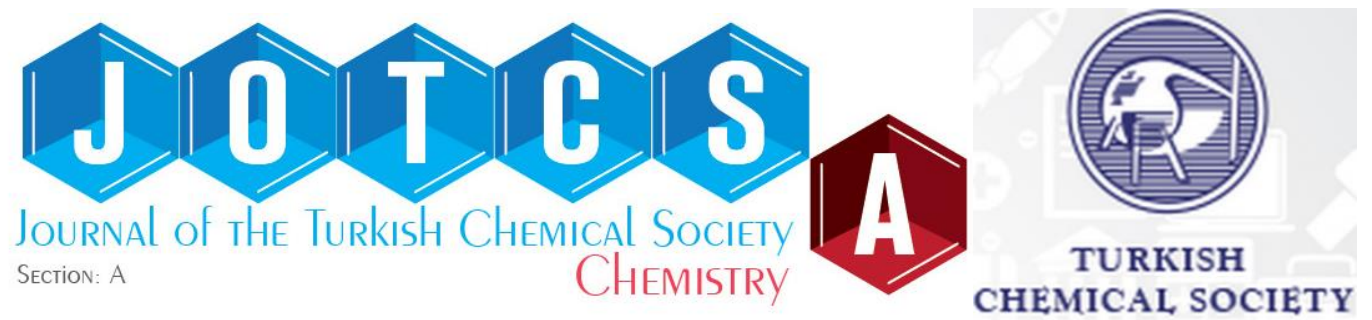

\title{
A New Approach To The Treatment of Leishmaniasis: Quercetin-Loaded Polycaprolactone Nanoparticles
}

\author{
Emrah Sefik Abamor 1 (D) \\ 1 Yildiz Technical University, Chemical and Metallurgical Engineering Faculty, Bioengineering Department, \\ Istanbul, Turkey
}

Abstract: Antileishmanial drugs used in the treatment of leishmaniasis are toxic and expensive. Moreover, parasites have recently developed resistance against them. Hence there is an increasing need for developing new antileishmanial medicines. Quercetin, found in the roots, leaves and fruits of many plants, is a natural polyphenolic flavonoid. Quercetin has antibacterial, antiviral, anti-carcinogenic, and antioxidant properties. On the other hand, because of its weak solubility in water, quercetin has had limited use on humans. To increase its bio-availability and maximize its therapeutic effects, quercetin has recently been encapsulated with nanoparticulate carrier systems. The aim of this study is to encapsulate quercetin in biodegradable, bio-compatible poly- $\varepsilon$-caprolactone (PCL) nanoparticles, to characterize the synthesized nanoparticles and to analyze their in vitro antileishmanial efficacy on L.infantum parasites. Quercetinloaded PCL nanoparticles (QPNPs) were synthesized using oil-in-water single emulsion solvent evaporation method. Their characterization was done using scanning electron microscopy (SEM) and dynamic light scattering (DLS) equipments. Encapsulation effectiveness and release profiles of QPNPs are calculated with UV-Vis spectrophotometry. The antileishmanial effectiveness of the synthesized nanoparticles was analyzed in L.infantum promastigote culture and amastigote-macrophage culture. The results indicated that QPNPS had an average size of $380 \mathrm{~nm}$, a zeta potential of $-6.56 \mathrm{mV}$, and a PDI value of 0.21 . The measurements showed the quercetin-loaded nanoparticles to have an encapsulation effectiveness of $64 \%$ and a reaction efficiency of $55 \%$. After an incubation of 192 hours, nanoparticles were seen to release $58 \%$ of their quercetin content. The synthesized QPNPs had IC50 values on L.infantum promastigotes and amastigotes of 86 and $144 \mu \mathrm{g} / \mathrm{mL}$ respectively. This means that QPNPs have reduced the vitality of promastigotes about 20 times and of amastigotes about 5 times as compared to the control group. These results demonstrate the strong antileishmanial potentials of QPNPS. It is believed that if these positive findings are supported by further in vivo studies, QPNPs may be used in the treatment of leishmaniasis.

Keywords: Leishmania, quercetin, polycaprolactone, nanoparticles, delivery.

Submitted: April 23, 2018. Accepted: September 05, 2018.

Cite this: Abamor E. A New Approach To The Treatment of Leishmaniasis: Quercetin-Loaded Polycaprolactone Nanoparticles. JOTCSA. 2018;5(3):1071-82.

DOI: $\underline{\text { http://dx.doi.org/10.18596/jotcsa.417831. }}$

*Corresponding author. E-mail: esabamor@gmail.com.

\section{INTRODUCTION}

Leishmaniasis disease, caused by the Leishmania parasites, which are among the obligate intracellular protozoans, is one of the most important health concerns of our country and the world (1). The disease has three different clinical forms, cutaneous, visceral, and mucocutaneous. Its most common form, Cutaneous Leishmaniasis $(\mathrm{CL})$, is a severe disease that causes the occurrence of single or multiple large lesions that can usually remain unhealed at the open areas of the body such as hands, face, arms, and legs. Another form of the disease, Visceral Leishmaniasis, causes hepatosplenomegaly in patients' visceral organs such as liver and spleen, and can have severe clinical manifestations that can lead to death if left untreated. Mucocutaneous Leishmaniasis is another form of the disease that mostly involves mucosal membranes and causes severe damage to organs such as mouth and nose, and sometimes leads to loss of the organ 
$(2,3)$. Currently, leishmaniasis is an endemic disease seen in 98 countries of the world, including Turkey. It is estimated that a total of 12 million people worldwide are infected with Leishmania parasites and about 300 million people at risk of developing the disease. According to the report published by the World Health Organization, it is estimated that a total of 1.5 million people develop $\mathrm{CL}$, while 500.000 people develop VL annually worldwide. According to the same report, it is estimated that each year, 60.000 people die due to the complications mediated by VL (4-7). Due to the fact that global warming and climate change increased their influence around the world, there is a concern that there would be an increase in the number of cases and mortality rate $(8,9)$. No vaccines with protective effect have been developed against the disease. Therefore, the only alternative in fighting against this disease is therapeutic practices. On the other hand, toxicity and the high cost of the drugs used in the treatment of leishmaniasis and the decrease in their effect with time, are the disadvantages of these drugs $(10,11)$. Moreover, in recent years, the parasites have developed resistance to anti-leishmania drugs (12). These conditions restrict the use of the existing antileishmania drugs in the treatment of the disease. Due to all these reasons, there is a significant need for the development of novel drug formulations to be used in the treatment of leishmaniasis.

In recent years, the use of herbal compounds in therapeutic practices is increasing rapidly (13). Quercetin is an important flavonoid that can be isolated from nearly 20 different plant species. Due to its antioxidant, anti-inflammatory, antibacterial, antiviral, antimutagenic, and anticarcinogenic properties, quercetin has been attracting the attention of especially pharmaceutical and food industries (14-16). Quercetin is considered as one of the best antioxidant flavonoids due to a large number of hydroxyl groups in its chemical structure (17). In contrast, due to its poor water solubility, inability to maintain its stability within the biological systems, and short half-life, the use of quercetin in clinical practices has been limited. Therefore, in order to increase the bioavailability of quercetin, use of the appropriate carrier systems is required $(18,19)$. Polycaprolactone $(P C L)$, is a biodegradable polyester synthesized by the ring opening polymerization of $\varepsilon$-caprolactone. $\mathrm{PCL}$ is degraded by the hydrolysis of its ester linkages under physiological conditions. Due to this property, PCL has been used in drug carrier systems as a biomaterial that allows long-term release. At the same time, as PCL is non-toxic, biocompatible and FDA-approved, the interest in the use of this polymer in clinical studies has increased. In most of the previous studies, it has been reported that the therapeutic efficiency of the molecules with low stability and solubility has increased upon their encapsulation by the $\mathrm{PCL}$ nanoparticles, and more efficient drug formulations are developed $(20,21)$. In one of these studies, Zheng et al. have encapsulated the drug named docetaxel, which is used in cancer treatment, in PCL-Tween80 copolymers, and characterized the resulting nanoparticles, and then investigated their anticarcinogenic efficiency in vitro on $\mathrm{C} 6$ glioma cancer cells. Based on the results, it was found that the nanoparticles, which were about $200 \mathrm{~nm}$ in diameter, were encapsulated by approximately $10 \%$, and made approximately $34 \%$ release within a 28 -day period. Results from in vitro experiments have shown that the nanoparticles loaded with the drug had a much higher anticarcinogenic effect than the application of the drug alone (22).

Until now, there have been studies showing the encapsulation of the quercetin molecule by many polymeric nanoparticle carrier systems, primarily PLGA. However, there are no studies in the literature on the encapsulation of quercetin by PCL nanoparticles and its antileishmanial activity. Considering the potential of quercetin to show a high antileishmanial activity despite its low stability and hydrophobic character, while the PCL is biodegradable, biocompatible and allows longterm drug release, we estimate that encapsulation of quercetin in PCL nanoparticles will eliminate the stability- and solubility-related disadvantages and have a strong antileishmanial activity on Leishmania parasites. Thus, the aim of this study is to investigate the synthesis and characterization of quercetin-loaded PCL nanoparticles (QPNPs) and to identify the in vitro antileishmanial activity on Leishmania promastigotes and amastigotes.

\section{EXPERIMENTAL SECTION}

\section{Materials}

PCL (MW:14.000), polyvinyl alcohol (PVA) (average MW: 30.000-70.000), Methylthiazolyltetrazolium (MTT) were purchased from Sigma-Aldrich (St. Louis, MO). Dichloromethane (DCM), sodium nitrite, sulfanylamide, naphthylethylenediamide dihydrochloride were obtained from Merck (Darmstadt, Germany). Roswell Park Memorial Institute medium (RPMI 1640) was purchased from GIBCO (Life Technologies, USA). Nigella sativa essential oil (Zade Vital) was commercially obtained from a national pharmacy. A mouse J774 macrophage cell line was obtained from the Histology and Embryology Department, Istanbul University, Istanbul, Turkey. Ultra-pure water was obtained from a Millipore MilliQ Gradient system.

\section{Preparation of Quercetin-Loaded PCL Nanoparticles}

Quercetin-loaded PCL nanoparticles (QPNPS) were prepared by an $\mathrm{o} / \mathrm{w}$ single solvent evaporation method. Briefly, $100 \mathrm{mg}$ of $\varepsilon$ caprolactone was dissolved in $5 \mathrm{~mL}$ of DCM. Then $10 \mathrm{mg}$ of quercetin was added into the organic phase. The organic phase was dropwise added 
into $25 \mathrm{~mL}$ of an aqueous phase including PVA (2 $\% \mathrm{w} / \mathrm{w})$ as a stabilizer. The mixture was emulsified for 5 minutes with a probe sonicator (Bandelin Sonopuls, Germany) at $80 \%$ amplitude in an ice bath. Evaporation of DCM from the emulsion was carried out by stirring at $750 \mathrm{rpm}$ for 4 h. Nanoparticle suspensions were centrifuged at $14,000 \mathrm{rpm}$ for 30 minutes. The pellet was rinsed twice with deionized distilled water. The obtained pellet was lyophilized for 48 $\mathrm{h}$ and stored at $-40^{\circ} \mathrm{C}$ until use.

\section{Characterization of nanoparticles}

Particle size, polydispersity index and zeta potential

Particle size, polydispersity index and zeta potential were identified by photon correlation spectroscopy (PCS) by using a Zetasizer Nano ZS (Malvern Instruments, Malvern, UK). Size measurements were performed in triplicate following preparation of nanoparticle suspensions by diluting in distilled water at a ratio of $1 / 100$ $(\mathrm{v} / \mathrm{v})$ at $25^{\circ} \mathrm{C}$. The polydispersity index range was evaluated at between 0 and 1 . The zeta potential analysis for synthetized nanoparticles was performed by using the same instrument at $25^{\circ} \mathrm{C}$.

\section{Scanning electron microscopy}

$$
\begin{aligned}
& \text { Encapsulation efficiency } \%=\frac{\text { Amount of encapsulated quercetin }}{\text { Initial amount of quercetin }} \times 100 \\
& \text { Reaction Yield } \%=\frac{\text { Amount of weighed (quercetin) PCL Nps }}{\text { Amount of initial quercetin and PCL }} \times 100
\end{aligned}
$$

\begin{abstract}
In vitro drug release studies
In order to provide release of quercetin, $5 \mathrm{mg}$ of quercetin encapsulated in PCL nanoparticles were suspended in $3 \mathrm{~mL}$ PBS at $\mathrm{pH}$ 7.4. Then suspension was incubated in a shaker incubator at $37^{\circ} \mathrm{C}$. At appropriate intervals, nanoparticles were centrifuged at $12,000 \mathrm{rpm}$ for $20 \mathrm{~min}$ and the supernatant was collected for the analysis. The amount of released quercetin within the supernatant was evaluated by UV spectrophotometer as described previously.
\end{abstract}

\section{Leishmania infantum Promastigote Culture} Leishmania infantum (L. infantum) promastigotes were cultivated in RPMI-1640 medium supplemented with $10 \%$ fetal bovine serum (FBS) at $27^{\circ} \mathrm{C}$. Metacyclic promastigotes were harvested at late log phase following to $120 \mathrm{~h}$ incubation.

\section{J774 Macrophage Cell Culture}

J774 macrophage cells were grown in RPMI 1640 medium supplemented with $100 \mathrm{U} / \mathrm{mL}$ penicillinstreptomycin and $10 \%$ FBS. Afterwards, macrophages were incubated at $37{ }^{\circ} \mathrm{C}$ incubator with $5 \% \mathrm{CO}_{2}$.

\section{Cell Viability Assay}

Cytotoxicity analysis of QPNPs and free nanoparticles were performed on $\mathrm{J774}$ macrophage cells. At first, $1 \times 10^{4}$ macrophage
Lyophilized nanoparticles were fixed on metallic studs like a thin film using adhesive tape and then coated with gold under vacuum. The particles were monitored by using an Evo LS10 (Zeiss, Welwyn Garden City, UK) scanning electron microscope (SEM) at enhanced voltage of 10-20 $\mathrm{kV}$.

\section{Encapsulation efficiency measurements}

The encapsulated amount of quercetin was determined by measuring the amount of quercetin remained in supernatant following to centrifugation process by using UV-Vis spectrophotometry at $374 \mathrm{~nm}$. The quantification was done in triplicate.

In order to determine non-encapsulated quercetin concentrations in the supernatant, a standard calibration curve of quercetin that was prepared at various concentrations was used. The concentration of encapsulated quercetin was ascertained by calculating the differences between initial concentrations of quercetin (10 $\mathrm{mg}$ ) and the concentration of free quercetin remained in the supernatant. The reaction yield (RY) and encapsulation efficiency (EE) of quercetin was calculated by using the following formulas: cells were seeded into each wells of 96-well plates and incubated for $24 \mathrm{~h}$ to allow cellular attachment. Then macrophages were exposed to free PCL nanoparticles and QPNPs at various concentrations ranging between 50 and 1000 $\mu \mathrm{g} / \mathrm{mL}$ for $144 \mathrm{~h}$. Cells that were not treated with neither free or loaded nanoparticles were identified as positive control. MTT test was used in order to detect cellular viabilities of macrophages after their exposure to different concentrations of formulations. For that purpose, $10 \mu \mathrm{L}$ of MTT reactant (Thiazolyl Blue Tetrazolium Bromide) $(10 \mathrm{mg} / \mathrm{mL})$ was included into each well and cells were then incubated for $4 \mathrm{~h}$. Following to incubation, DMSO was put into each wells in order to dissolve the formazan crystals. Absorbance values was measured at $570 \mathrm{~nm}$ using a microplate reader (Thermo Scientific, Multiskan FC).

\section{Anti-promastigote Assay}

Antileishmanial activities of quercetin-loaded PCL nanoparticles and free nanoparticles were performed on $L$. infantum promastigotes and amastigotes, in vitro. For anti-promastigote assay, $5 \times 10^{5} \mathrm{~L}$. infantum promastigotes were transfered into a 6-well plate and cells were incubated at $27^{\circ} \mathrm{C}$ for $24 \mathrm{~h}$. Afterwards, various concentrations of quercetin-encapsulated $\mathrm{PCL}$ nanoparticles and free nanoparticles varying between 50 and $1000 \mu \mathrm{g} / \mathrm{mL}$ was added into 
wells. Treated promastigotes were incubated at $27{ }^{\circ} \mathrm{C}$ for $192 \mathrm{~h}$. The number of viable promastigotes for each groups were counted with hemocytometer at the end of 96 and $192 \mathrm{~h}$ incubation period. For that purpose, a $50-\mu \mathrm{L} L$. infantum promastigote culture that was taken from each well was fixed with $2 \%$ formalin at a ratio of $1: 10$. Afterwards, suspensions were transferred into a hemocytometer, and the slide was investigated in an inverted microscope (Olympos CKX41). IC50 values of loaded and empty nanoparticles were determined by evaluating the concentration that inhibited half of L. infantum promastigotes.

\section{Determination of Anti-amastigote Efficacies}

Studies on determination of anti-amastigote effects of quercetin loaded and free nanoparticles were performed on amastigote-macrophage culture. For that purpose, $2.5 \times 10^{4} \mathrm{J774}$ macrophage cells were seeded into 6 -well plates and incubated at $37^{\circ} \mathrm{C}$. Following to $24 \mathrm{~h}$ incubation, $2.5 \times 10^{5} \mathrm{~L}$. infantum promastigotes were added into each well in order to provide infection of macrophage cells by parasites. After $4 \mathrm{~h}$ incubation at $37^{\circ} \mathrm{C}$, non-phagocytized promastigotes were removed by washing the plates triplicate with PBS. Thus, amastigotemacrophage culture was established. After that, various concentrations of quercetin loaded PCL nanoparticles and free nanoparticles ranginig between 50 and $1000 \mu \mathrm{g} / \mathrm{mL}$ were included into 6 -well plates in order to find efficacies of nanoparticles on $L$. infantum amastigotes.
Treated amastigote-macrophage culture was incubated at $37^{\circ} \mathrm{C}$ for $192 \mathrm{~h}$. Following to incubation, all wells were rinsed three times with sterile PBS and then fixed with methanol for 10 min. Afterwards, each well was stained with giemsa for $3 \mathrm{~min}$ and was washed with PBS following incubation. Then, slides that were inserted into each well were removed and investigated on inverted microscope at $100 \mathrm{X}$ dimensions. The infection index of all samples were evaluated by multiplying \% infectivity values with amastigote number per macrophages. \% infectivity was assessed by dividing the number of infected macrophages with the number of all macrophages in the zone.

\section{RESULTS}

\section{Characterization of synthetized} nanoparticles

Characterization of the QPNPs and empty PCL nanoparticles was performed using scanning electron microscope and Zetasizer equipment. At the same time, the reaction yields and encapsulation practices of the quercetin-loaded nanoparticles were calculated and nanoparticles were characterized. In Figure 1, a view of the SEM analysis of QPNPs is provided. As it can be seen from the image, the nanoparticles that are synthesized are round, uniform, and smooth. At the same time, the nanoparticles are close to each other in size. Their sizes vary between 200$400 \mathrm{~nm}$.

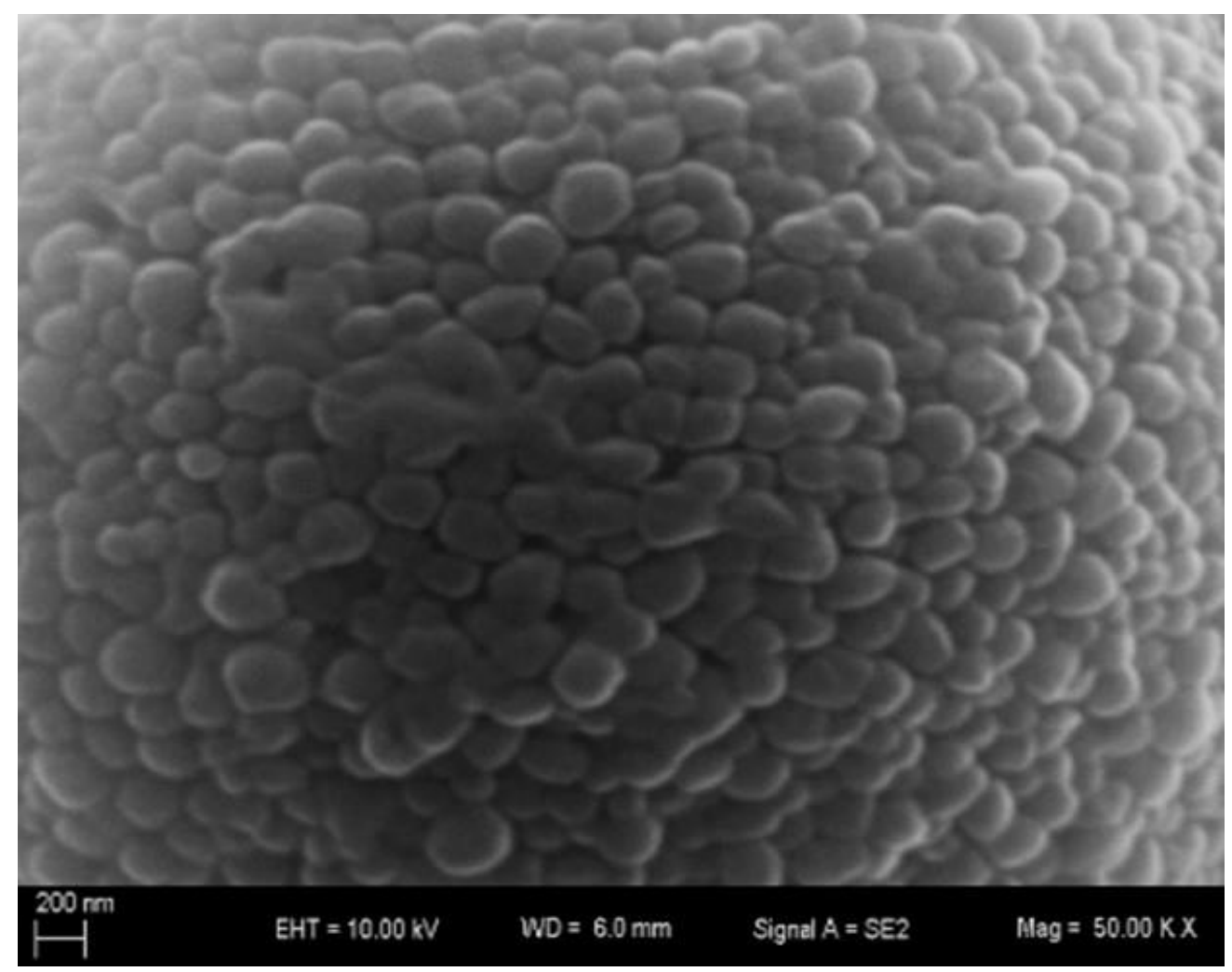

Figure 1. SEM image of quercetin encapsulated PCL nanoparticles.

The result of the size analysis performed by Zetasizer has shown that the empty nanoparticles have an average size of $220 \mathrm{~nm}$, while QPNPs have an average size of $350 \mathrm{~nm}$ (Table 1 ). The 
distribution of QPNPS due to their sizes as a result of Dynamic Light Scattering (DLS) analysis was also shown in Figure 2. It is estimated that the difference in size between the empty and loaded nanoparticles can be due to the encapsulation of quercetin by the PCL nanoparticles. On the other hand, polydispersity index (PDI) values, zeta potentials, encapsulation efficiencies and reaction yield of the empty and loaded nanoparticles are given in Table 1 . As can be seen from the table, the zeta potentials of the empty nanoparticles and QPNPs are -4.92 and -6.56 , respectively.
Negative values of the zeta potentials are due to the carboxyl end groups of the PCL polymers.

When PDI values of the empty and quercetinloaded nanoparticles are analyzed, it was found that this value varied between 0.11 and 0.21 for the empty nanoparticle. These results show that the synthesized nanoparticles are close to each other in terms of size and are distributed homogeneously. In Table 1, it was shown that for the quercetin-loaded nanoparticles, the encapsulation efficiency is $64 \%$ and the reaction yield is $55 \%$.

Table 1. Demonstration of mean size, PDI values, zeta potential measurements, encapsulation efficiency and reaction yield percentages of free nanoparticles and quercetin-loaded PCL nanoparticles.

\begin{tabular}{cccccc}
\hline Formulations & Size $\mathbf{( n m )}$ & PDI & $\begin{array}{c}\text { Zeta } \\
\text { Potential } \\
\mathbf{( m V )}\end{array}$ & $\begin{array}{c}\text { Encapsulation } \\
\text { Efficiency } \\
\mathbf{( \% )}\end{array}$ & $\begin{array}{c}\text { Reaction } \\
\text { Yield (\%) }\end{array}$ \\
\hline Free NPs & 220 & 0,12 & $-4,92 \pm 0,63$ & - & - \\
QPNPs & 350 & 0,21 & $-6.56 \pm 0,45$ & 64 & 55 \\
\hline
\end{tabular}

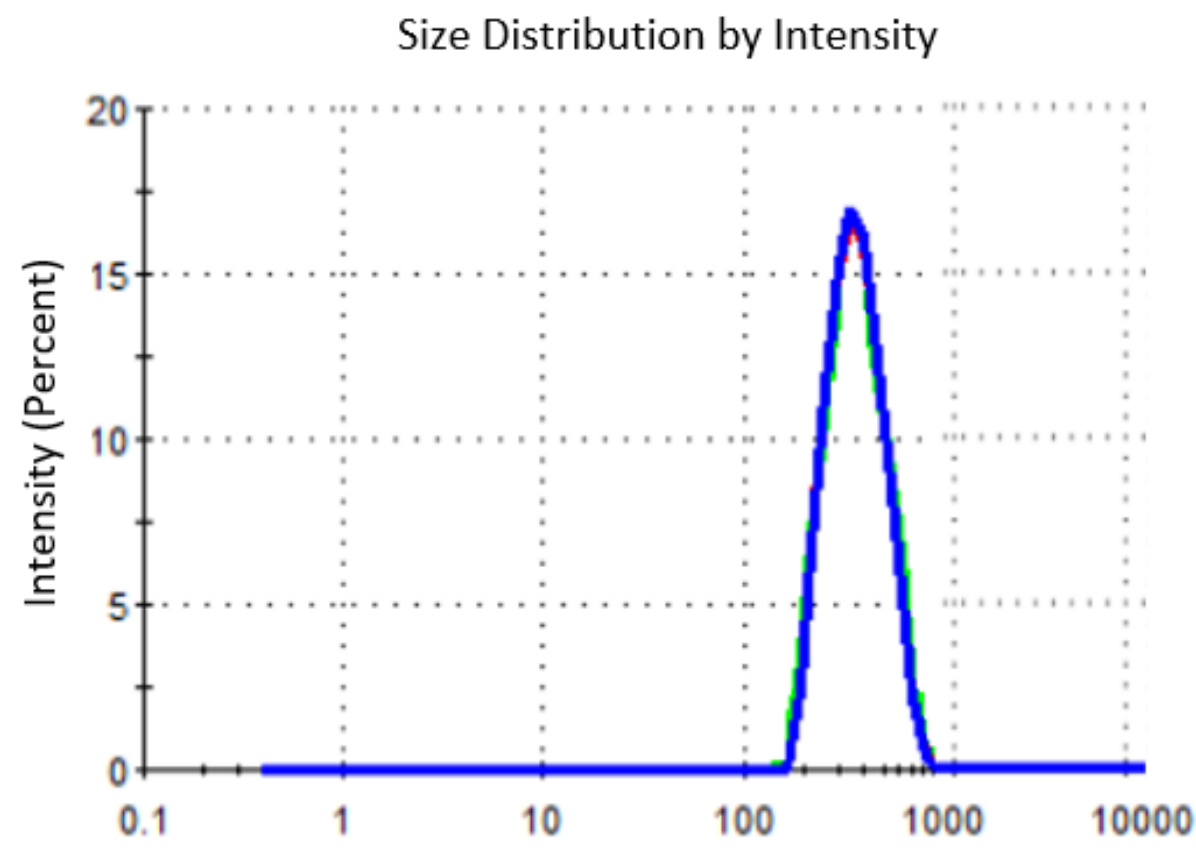

Figure 2. Size distribution analysis of quercetin loaded PCL nanoparticles by DLS.

\section{In vitro Release Study}

In order to determine the in vitro release of quercetin from the nanoparticle formulations, a dissolution method performed at $\mathrm{pH} 7.4$ was used. $240 \mathrm{~h}$ cumulative release profiles of the quercetin-loaded nanoparticles are shown in Figure 3. As it can be seen from the graph, within the first $4 \mathrm{~h}$, drug-loaded nanoparticles had a $9 \%$ initial burst effect. The amount of quercetin released from the nanoparticles until the $144^{\text {th }}$ hour of the incubation increased every day. At the end of the $144^{\text {th }}$ hour, the total amount of quercetin released from the PCL nanoparticles was $2.2 \mathrm{mg}$. This shows that the nanoparticles release approximately $32 \%$ of their quercetin load at the end of the 144-hour-long incubation. The release study was continued for 240 hours. At the end of 240 hours, it was found that the nanoparticles released approximately $58 \%$ quercetin. The cumulative amount of quercetin released from the beginning until the $240^{\text {th }} \mathrm{h}$ was calculated as $3.6 \mathrm{mg}$. 


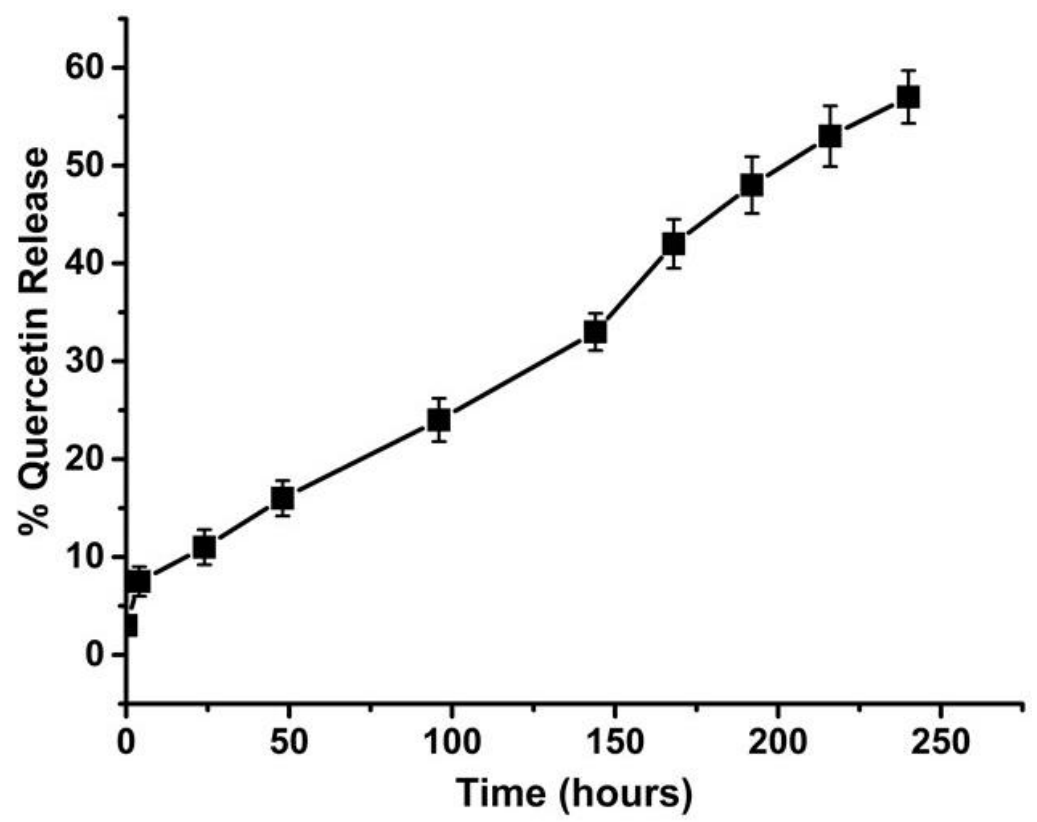

Figure 3. In vitro release profile of quercetin-encapsulated PCL nanoparticles after 240 hours of incubation.

\section{Cytotoxicity Analysis}

Cytotoxicity analysis for different concentrations of quercetin, quercetin-loaded PCL nanoparticles, and empty nanoparticles was performed using 3774 cells. As it can be seen in Figure 4, the toxic effects of empty nanoparticles and quercetinloaded nanoparticles on $\mathbf{3 7 7 4}$ cells are quite low. In particular, it was found that the vitality of these cells exposed to these nanoparticles at low concentrations is nearly the same with the control. The vitality rates of the cells exposed to the highest concentration $(1000 \mu \mathrm{g} / \mathrm{mL})$ of empty nanoparticles and QPNPs were found to be \%90 and \%84, respectively. On the other hand, cytotoxic effects of quercetin on cells was found to be higher compared to the other groups. It was found that the cells exposed to $1000 \mu \mathrm{g} / \mathrm{mL}$ quercetin had their vitality reduced to $66 \%$.

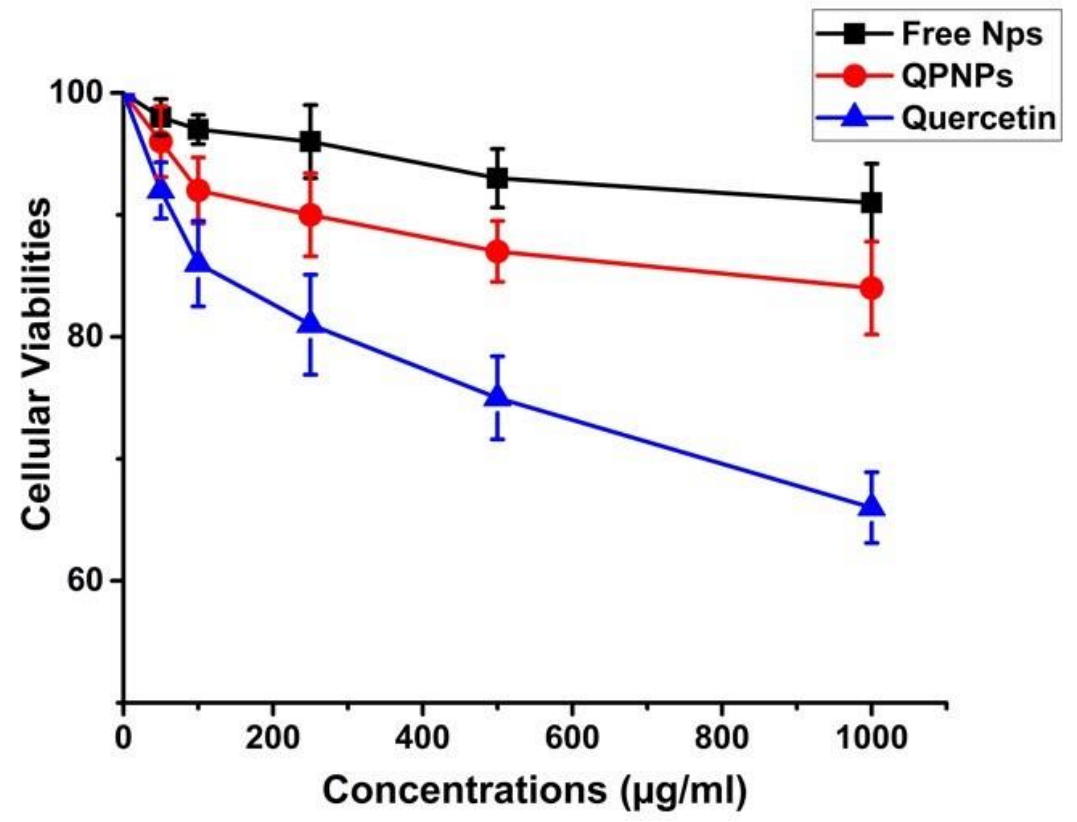

Figure 4. Cellular viability ratios of macrophage cells that were exposed to different concentrations of free NPs, QPNPs and quercetin, respectively.

Identification of the Anti-Promastigotic Activity

In the study, the activities of quercetin alone, quercetin-loaded PCL nanoparticles, and empty PCL nanoparticles on $L$. infantum promastigotes were studied using the measurements taken at the $192^{\text {nd }}$ hour of the incubation. As can be seen from Figure 5, it was found that quercetin alone and QPNPs show high levels of antileishmanial activity on $L$. infantum promastigotes. However, empty nanoparticles have very low antileishmanial activity on $L$. infantum parasites. The activities of quercetin and QPNPs increase depending on the concentration. It was found that 
while the vitality rate of $L$. infantum parasites exposed to quercetin at a $50 \mu \mathrm{g} / \mathrm{mL}$ concentration was $77 \%$, the vitality of those exposed to 1000 $\mu \mathrm{g} / \mathrm{mL}$ decreased to $20 \%$ (Figure 5). On the other hand, it was found that QPNPs have a higher antileishmanial activity compared to the quercetin alone. While the vitality rate of $L$. infantum parasites exposed to $50 \mu \mathrm{g} / \mathrm{mL}$ QPNPs was $69 \%$, this ratio decreased gradually depending on the increase in the concentration and the vitality finally decreased to $8 \%$ for the $L$. infantum promastigotes exposed to $1000 \mu \mathrm{g} / \mathrm{mL}$ quercetin. On the other hand, it was found that the vitality rate was around $80 \%$ for the group exposed to the empty nanoparticles at the highest concentration. Upon analyzing the IC50 values of the formulations, it was found that these values were $86 \mu \mathrm{g} / \mathrm{mL}, 149 \mu \mathrm{g} / \mathrm{mL}$, and $>1000 \mu \mathrm{g} / \mathrm{mL}$ for quercetin-loaded $\mathrm{PCL}$ nanoparticles, quercetin, and empty nanoparticles, respectively. As can be seen, among the formulations, QPNPS had the highest activity. The activity of quercetin on parasites increased after being encapsulated in the PCL nanoparticles. On the other hand, it was found that the empty nanoparticles did not have a significant effect on parasites.

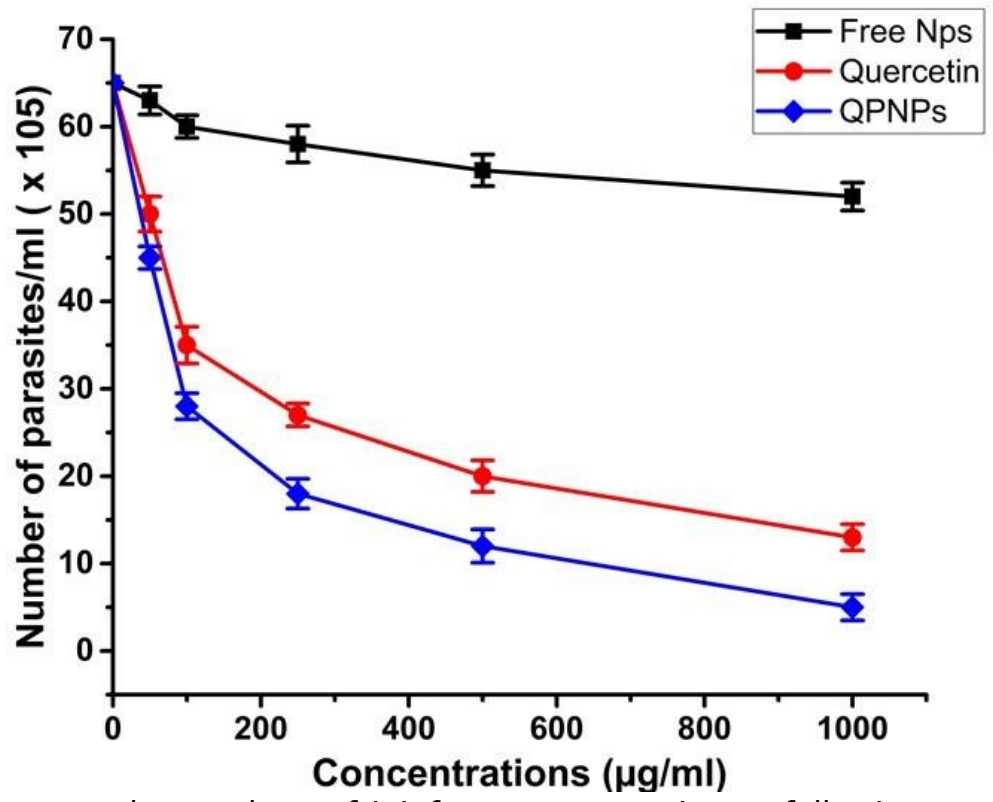

Figure 5. The decrease on the numbers of L.infantum promastigotes following to exposure to different concentrations of free NPs, QPNPs and quercetin for $192 \mathrm{~h}$.

\section{Identification of the Anti-Amastigotic Activity}

In order to investigate the activities of quercetin alone, empty, and QPNPs on $L$. infantum amastigotes, infection indices of the infected macrophages on which the formulations were applied were calculated. Compared to the control group on which no formulations were applied, the change in the infection indices of the macrophages in the experimental groups revealed whether the formulations had a concentration-dependent effect on amastigotes. Infection index of the macrophages in the control group was 205. In the experimental group on which empty nanoparticles were applied, it was found that the infection index varied between 192 and 169 depending on the concentration (Figure $6)$. As can be seen, empty nanoparticles did not have a significant effect on $L$. infantum amastigotes. In contrast, the anti-amastigotic activity of quercetin alone and QPNPs were higher than the control and empty nanoparticle groups.
Infection indices of the macrophages exposed to quercetin alone varied between 164 and 69 . Infection indices of the macrophages exposed to $50 \mu \mathrm{g} / \mathrm{mL}$ quercetin were 164 , whereas that of the macrophages exposed to $1000 \mu \mathrm{g} / \mathrm{mL}$ quercetin was 69. IC50 value of quercetin on $L$. infantum amastigotes was $300 \mu \mathrm{g} / \mathrm{mL}$. It was found that QPNPs have a higher anti-amastigotic activity compared to the application of quercetin alone. While the infection index of the macrophages exposed to $50 \mu \mathrm{g} / \mathrm{mL}$ QPNPs was 146 , that of the macrophages exposed to 1000 $\mu \mathrm{g} / \mathrm{mL}$ was 45 . As can be seen, compared to the control group, QPNPs decreased Leishmania amastigotes by 5 fold when used at the highest concentration. The IC50 value of the QPNPS on $L$. infantum amastigotes was calculated as 144 $\mu \mathrm{g} / \mathrm{mL}$. These results suggest that quercetinloaded nanoparticles have a 2 -fold higher antiamasigotic activity compared to the quercetin alone. 


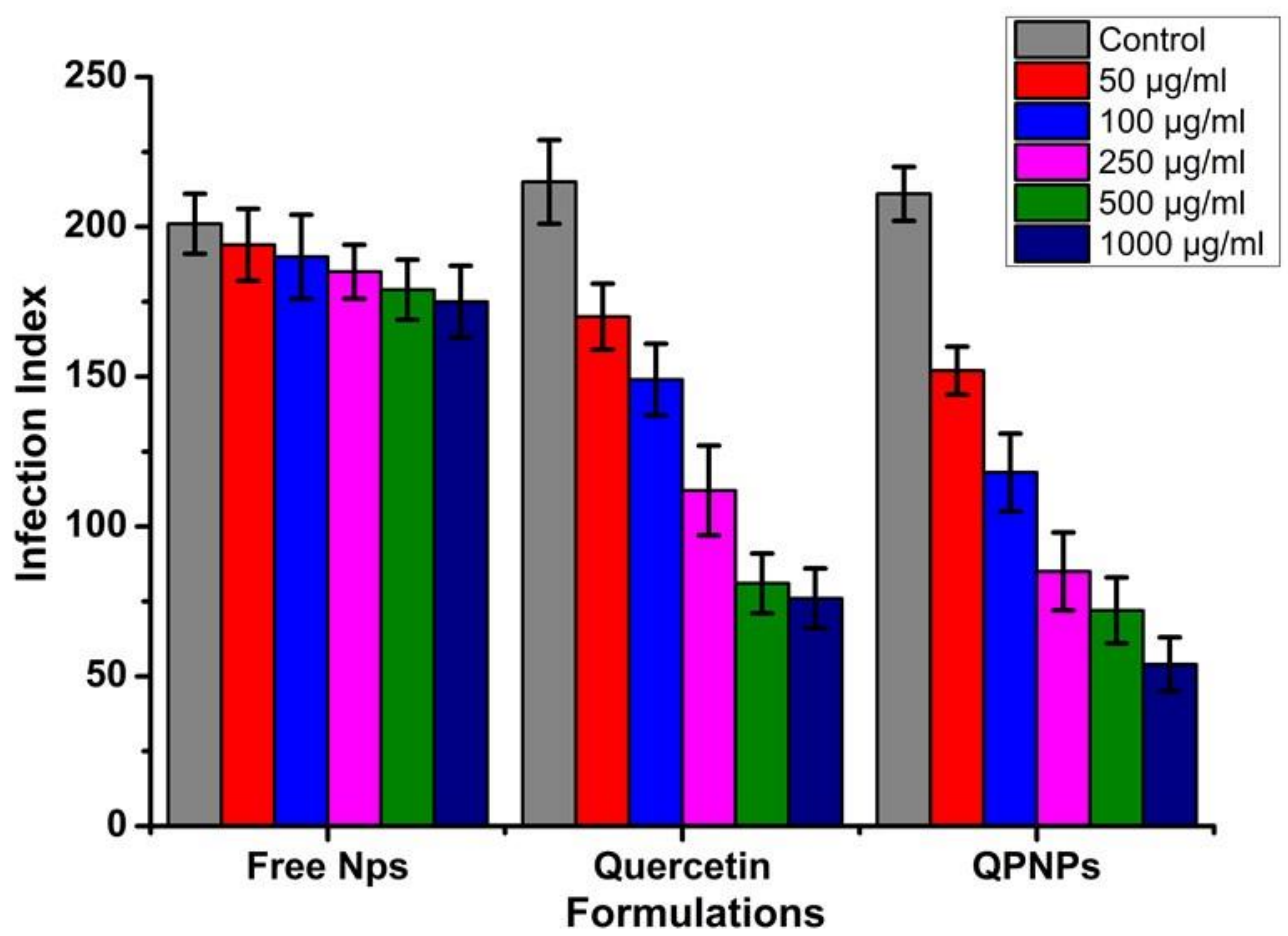

Figure 6. Infection index values of infected macrophages that were exposed to different concentrations of free NPs, QPNPs and quercetin for $192 \mathrm{~h}$.

\section{DISCUSSION}

Currently used anti-leishmanial drugs have various disadvantages such as toxicity, resistance, and high cost. Thus, there have been studies toward the development of new generation antileishmanial compounds in the recent years (23). Quercetin molecule, which is obtained from various plants, is one of the strongest known flavonoids. Anti-microbial, antioxidant, anti-carcinogenic and anti-inflammatory effects of quercetin have been proven in the previous studies (24-26). However, low water solubility of this molecule reduces its applicability in clinical practices. Therefore, encapsulation of quercetin by carrier systems in order to increase its solubility, bio-availability and therapeutic efficiency has been considered as an appropriate method. In this study, we encapsulated quercetin molecule in PCL nanoparticles and investigated the antileishmanial activities of the formulations on L. infantum promastigotes and amastigotes in vitro.

Encapsulation of quercetin by PCL nanoparticles was performed using oil-in-water single emulsion solvent evaporation technique. In the study by Arasoglu et al., this technique was shown to be more successful than nano-precipitation and salting out techniques used in the encapsulation of quercetin by PLGA nanoparticles. In the same study, it was found that the nanoparticles synthesized using single emulsion solvent evaporation have higher reaction yield and have a more significant antibacterial activity compared to the nanoparticles synthesized by other techniques (27). Size analysis and other characterization tests of the nanoparticles we prepared using oil-in-water single emulsion solvent evaporation technique were performed using SEM and zetasizer devices. According to the zetasizer analysis, the average size of empty nanoparticles was approximately $220 \mathrm{~nm}$, and the average size of quercetin-loaded nanoparticles was approximately $350 \mathrm{~nm}$. The larger size of the quercetin-loaded nanoparticles compared to the empty nanoparticles can be considered as an indicator of the efficient and effective encapsulation of quercetin by the $\mathrm{PCL}$ nanoparticles. In the previous studies, it was stated that the drug-loaded nanoparticles can be larger in size compared to the empty nanoparticles $(28,29)$. It was found that, particularly in cancer research, carrier systems smaller than $500 \mathrm{~nm}$ are more successful in delivering the drugs to the target tissues, therefore enabling active transport (30). On the other hand, it is known that nanoparticles larger than $200 \mathrm{~nm}$ are more effective in targeting macrophages that are used as host cells by the parasites, and that the nanoparticles of this size are phagocytosed by the macrophages (31). Thus, due to their size, it is estimated that the nanoparticles obtained in our study can directly target the parasites living inside the macrophages. Negative zeta potentials of the synthesized nanoparticles can be completely correlated with the negatively charged carboxyl groups of the used PCL polymer. Low PDI values is an indicator that the synthesized nanoparticles are close to each other in size. Based on the uptake analysis, it was found that following 240 hours of incubation, PCL nanoparticles release approximately $58 \%$ of the quercetin they contain into the environment. As known, PCL nanoparticles decompose more slowly than the 
other polymeric nanoparticles used in the drug carrier systems, especially PLGA. The reasons behind this are that PCL has a higher molecular weight and is more hydrophobic (32). In a study similar to the one we performed, Pathak et al. encapsulated the antibiotics doxycycline and metronidazole in PCL nanoparticles and investigated the activity of the synthesized nanoparticles in the treatment of intravaginal inflammatory diseases. In the release analysis performed within the context of the said study, it was found that the synthesized nanoparticles release approximately $60-70 \%$ of the drug molecules they contain at the end of the incubation period of 14 days. These results corroborate the results we obtained in our study (33).

Following the synthesis and characterization, we investigated the in vitro antileishmanial activities of the QPNPs we synthesized on $L$. infantum promastigotes and amastigotes. Antileishmanial activity of quercetin, one of the strongest known flavonoids, has been shown in previous studies. In a study by Belkhelfa-Slimani et al. in 2016, the apoptotic effects and cytotoxic activities of caffeic acid and quercetin on Leishmania major promastigotes were studied. In that study, it was found that caffeic acid and quercetin at a concentration of $400 \mu \mathrm{mol} / \mathrm{L}$ decrease the vitality of $L$. major parasites by $68.90 \%$ and $59.22 \%$, respectively. In the same study, it was shown that quercetin leads to caspase-independent apoptosis in parasites and cause cell death (34). The antileishmanial activity of quercetin is thought to be related to the production of reactive oxygen species (ROS), of which parasites are highly sensitive, causing damage to the mitochondria and cell membranes of parasites and inhibiting the nucleic acid synthesis. Due to these properties, quercetin and quercetincontaining extracts are known as strong antiparasitic agents $(35,36)$. However, as discussed earlier, low water solubility of quercetin is one of the most significant obstacles to the development of quercetin-based drug molecules. In recent years, studies have been performed toward the production of quercetin-loaded polymeric carrier systems to eliminate this obstacle and increase the therapeutic activity of quercetin. In these studies, PLGA nanoparticles are frequently used. Arasoglu et al. have investigated the antibacterial activities of the quercetin-loaded PLGA nanoparticles prepared using various synthesis methods on foodborne pathogens such as Listeria monocytogenes, Salmonella typhimurium, Escherichia coli, and Staphylococcus aureus. While quercetin and quercetin-loaded PLGA nanoparticles showed nearly similar levels of antibacterial activity on Salmonella typhimurium, Escherichia coli, and Staphylococcus aureus, quercetin-loaded PLGA nanoparticles showed higher level of antibacterial activity on Listeria monocytogenes. The MIC value of quercetin-loaded PLGA nanoparticles on these bacteria was $100 \mu \mathrm{g} / \mathrm{mL}$ whereas the MIC value of quercetin alone was $200 \mu \mathrm{g} / \mathrm{mL}$ (27). In a similar study from 2016, Sun et al. investigated the in vitro and in vivo antibacterial effects of quercetin-loaded PLGA nanoparticles on $E$. coli and Micrococcus tetragenus bacteria. Unlike the other study, quercetin-loaded nanoparticles had a higher level of antibacterial activity compared to the quercetin alone. Based on the results, the vitality of the $E$. coli exposed to $70 \mu \mathrm{L}$ quercetinloaded PLGA nanoparticle decreased by $92 \%$ whereas this rate was $66 \%$ in the $E$. coli exposed to quercetin alone. Similarly, the vitality of the $M$. tetragenus exposed to the nanoparticles loaded with the same dose was calculated as $26 \%$ and the vitality of the bacteria exposed to quercetin was calculated as $49 \%$. Based on these results, it can be said that quercetin and quercetin-loaded PLGA nanoparticles have a higher antimicrobial effect on $E$. coli than on M. tetragenus. Moreover, it was found that the therapeutic activity of quercetin increases upon its encapsulation by the PLGA nanoparticles (37). On the other hand, so far no studies have been performed on the encapsulation of the quercetin by the biocompatible, biodegradable, FDA-approved polymer $\mathrm{PCL}$ and its antileishmanial activities. In the present study, in vitro antileishmanial activities of the QPNPs on $L$. infantum promastigotes and amastigotes are investigated for the first time. Based on the results, the IC50 values of the QPNPs on $L$. infantum promastigotes and amastigotes were calculated as 86 and 144 $\mu \mathrm{g} / \mathrm{mL}$, respectively. On the other hand, the IC50 values of quercetin on $L$. infantum promastigotes and amastigotes were calculated as 149 and 300 $\mu \mathrm{g} / \mathrm{mL}$. These results indicate that the antileishmanial activity of the quercetin encapsulated in the PCL nanoparticles increases significantly. At the same time, it was found that QPNPs are more effective on $L$. infantum promastigotes than on amastigotes. Since the nanoparticles should be penetrated inside the macrophages in order to contact with the amastigotes, their efficiency on amastigotes can be lower. It has also been emphasized in previous studies that in order for the drug-loaded nanoparticles to penetrate in the macrophages more efficiently and inhibit amastigotes, they should be applied at higher concentrations than the concentrations required for the promastigotes. On the other hand, it was found that QPNPs inhibited $78 \%$ of the amastigotes when used at the highest concentration after 192 hours of incubation. Considering that nanoparticles only allow 50\% quercetin release within that period, it is estimated that after longer incubation periods, more of the quercetin can be released, and thus, nearly all of the amastigotes can be inhibited.

In conclusion, within the context of this study, encapsulation of the quercetin in $P C L$ nanoparticles has been performed with success for the first time, and the significant antileishmanial activity of the QPNPs on $L$. infantum promastigotes and amastigotes has 
been observed. These promising data suggest that QPNPs can be successful in the eradication of leishmaniasis. In case of obtaining positive results in the in vivo studies, QPNPs can be used in the treatment of leishmaniasis.

\section{REFERENCES}

1. Vannier-Santos MA, Martiny A, de Souza W. Cell biology of Leishmania spp.: invading and evading. Curr Pharm Des. 2002;8(4):297-318.

2. de Vries HJ, Reedijk SH, Schallig HD.

Cutaneous leishmaniasis: recent developments in diagnosis and management. Am J Clin Dermatol. 2015;16(2):99-109.

3. Bailey MS, Lockwood DN. Cutaneous leishmaniasis. Clin Dermatol. 2007;25(2):20311.

4. Torres-Guerrero E, Quintanilla-Cedillo MR, Ruiz-Esmenjaud J, Arenas R. Leishmaniasis: a review. F1000Res. 2017;6:750.

5. Reithinger R, Dujardin JC, Louzir H, Pirmez C, Alexander B, Brooker S.Cutaneous leishmaniasis. Lancet Infect Dis. 2007;7(9):58196.

6. Bravo F, Sanchez MR. New and re-emerging cutaneous infectious diseases in Latin America and other geographic areas. Dermatol Clin. $2003 ; 21(4): 655-68$

7. Gurunath U, Joshi R, Agrawal A, Shah V. An overview of visceral leishmaniasis elimination program in India: a picture imperfect. Expert Rev Anti Infect Ther.2014;12(8):929-35.

8. González C, Wang O, Strutz SE, GonzálezSalazar C, Sánchez-Cordero V, Sarkar S. Climate change and risk of leishmaniasis in north america: predictions from ecological niche models of vector and reservoir species. PLoS Negl Trop Dis. 2010;4(1):e585.

9. Stamm LV. Human Migration and Leishmaniasis-On the Move. JAMA Dermatol. 2016;152(4):373-4.

10. Singh OP, Singh B, Chakravarty J, Sundar S. Current challenges in treatment options for visceral leishmaniasis in India: a public health perspective. Infect Dis Poverty. 2016;5:19.

11. Moore EM, Lockwood DN. Treatment of visceral leishmaniasis. J Glob Infect Dis. 2010;2(2):151-8.

12. Chakravarty J, Sundar S. Drug resistance in leishmaniasis. J Glob Infect Dis. 2010;2(2):16776.

13. Ekor $M$. The growing use of herbal medicines: issues relating to adverse reactions and challenges in monitoring safety. Front Pharmacol. 2014;4:177.

14. Anand David AV, Arulmoli R, Parasuraman S. Overviews of Biological Importance of Quercetin: A Bioactive Flavonoid. Pharmacogn Rev. 2016;10(20):84-89.

15. Salvamani S, Gunasekaran B, Shaharuddin NA, Ahmad SA, Shukor MY. Antiartherosclerotic effects of plant flavonoids. Biomed Res Int. $2014 ; 2014: 480258$.

16. Sultana B, Anwar F. Flavonols (kaempeferol, quercetin, myricetin) contents of selected fruits, vegetables and medicinal plants. Food Chem. 2008;108(3):879-84.

17. Moalin $M$, Strijdonck GP, Beckers $M$, Hagemen G, Borm P, Bast A, Haenen GR. A planar conformation and the hydroxyl groups in the $B$ and $C$ rings play a pivotal role in the antioxidant capacity of quercetin and quercetin derivatives. Molecules. 2011;16(11):9636-50.

18. Kumari A, Yadav SK, Pakade YB, Singh B, Yadav SC. Development of biodegradable nanoparticles for delivery of quercetin. Colloids Surf B Biointerfaces. 2010;80(2):184-92.

19. Natarajan V, Krithica N, Madhan B, Sehgal PK. Formulation and evaluation of quercetin polycaprolactone microspheres for the treatment of rheumatoid arthritis. J Pharm Sci. 2011 Jan;100(1):195-205.

20. McNeil SE, Griffiths HR, Perrie Y. Polycaprolactone fibres as a potential delivery system for collagen to support bone regeneration. Curr Drug Deliv. 2011;8(4):448-55.

21. Madhaiyan K, Sridhar R, Sundarrajan S, Venugopal JR, Ramakrishna S. Vitamin B12 loaded polycaprolactone nanofibers: a novel transdermal route for the water soluble energy supplement delivery. Int J Pharm. 2013 Feb 28;444(1-2):70-6.

22. Ma $Y$, Zheng $Y$, Zeng $X$, Jiang $L$, Chen $H$, Liu R, Huang L, Mei L. Novel docetaxel-loaded nanoparticles based on PCL-Tween 80 copolymer for cancer treatment. Int J Nanomedicine. 2011;6:2679-88.

23. de Menezes JP, Guedes CE, Petersen AL, Fraga DB, Veras PS. Advances in Development of New Treatment for Leishmaniasis. Biomed Res Int. $2015 ; 2015: 815023$.

24. Nam JS, Sharma AR, Nguyen LT, Chakraborty C, Sharma G, Lee SS. Application of Bioactive Quercetin in Oncotherapy: From Nutrition to Nanomedicine. Molecules. 2016;21(1):E108. 
25. Johari J, Kianmehr A, Mustafa MR, Abubakar $S$, Zandi K. Antiviral activity of baicalein and quercetin against the Japanese encephalitis virus. Int J Mol Sci.2012;13(12):16785-95.

26. Ramos FA, Takaishi $Y$, Shirotori $M$, Kawaguchi Y, Tsuchiya K, Shibata H, Higuti T, Tadokoro T, Takeuchi M. Antibacterial and antioxidant activities of quercetin oxidation products from yellow onion (Allium cepa) skin. J Agric Food Chem. 2006;54(10):3551-7.

27. Arasoglu T, Derman S, Mansuroglu B, Uzunoglu D, Kocyigit B, Gumus B, Acar T, Tuncer $B$. Preparation, characterization, and enhanced antimicrobial activity: quercetinloaded PLGA nanoparticles against foodborne pathogens. Turk J Biol. 2017;41:p127-140.

28. Zheng D, Li X, Xu H, Lu X, Hu Y, Fan W. Study on docetaxel-loaded nanoparticles with high antitumor efficacy against malignant melanoma. Acta Biochim Biophys Sin (Shanghai). 2009 Jul;41(7):578-587

29. Rachmawati $H$, Yanda $Y L$, Rahma A, Mase N. Curcumin-Loaded PLA Nanoparticles: Formulation and Physical Evaluation. Sci Pharm. 2016 Feb $14 ; 84(1): 191-202$.

30. Li B, Li Q, Mo J, Dai H. Drug-Loaded Polymeric Nanoparticles for Cancer Stem Cell Targeting. Front Pharmacol. 2017 Feb 14;8:51.

31. Kalluru R, Fenaroli $F$, Westmoreland $D$, Ulanova L, Maleki A, Roos N, Paulsen Madsen M, Koster G, Egge-Jacobsen W, Wilson S, RobergLarsen H, Khuller GK, Singh A, Nyström B, Griffiths G. Poly(lactide-co-glycolide)-rifampicin nanoparticles efficiently clear Mycobacterium bovis BCG infection in macrophages and remain membrane-bound in phago-lysosomes. J Cell Sci. $2013 ; 126(14): 3043-54$.

32. Danafar H, Schumacher U. MPEG-PCL copolymeric nanoparticles in drug delivery systems. Cogent Medicine. 2016;3:1

33. Pathak M, Coombes AGA, Ryu B, Cabot PJ, Turner MS, Palmer C, Wang D, Steadman KJ. Sustained Simultaneous Delivery of Metronidazole and Doxycycline From Polycaprolactone Matrices Designed for Intravaginal Treatment of Pelvic Inflammatory Disease. J Pharm Sci. 2018;107(3):863-869.

34. Belkhelfa-Slimani R, Djerdjouri B. Caffeic acid and quercetin exert caspases-independent apoptotic effects on Leishmania major promastigotes, and reactivate the death of infected phagocytes derived from BALB/c mice. Asian Pacific Journal of Tropical Biomedicine. 2017;7(4):321-331

35. Fonseca-Silva F, Inacio JD, Canto-Cavalheiro MM, Almeida-Amaral EE. Reactive oxygen species production and mitochondrial dysfunction contribute to quercetin induced death in Leishmania amazonensis. PLoS One. $2011 ; 6(2): e 14666$.

36. Kheirandish $F$, Delfan $B$, Mahmoudvand $H$, Moradi N, Ezatpour B, Ebrahimzadeh F, Rashidipour M. Antileishmanial, antioxidant, and cytotoxic activities of Quercus infectoria Olivier extract. Biomed Pharmacother. 2016;82:208-15.

37. Sun D, Li N, Zhang W, Yang E, Mou Z, Zhao Z, Liu H, Wang W. Quercetin-loaded PLGA nanoparticles: a highly effective antibacterial agent in vitro and anti-infection application in vivo. J Nanopart Res. 2016;18:1-21. 
Abamor EŞ. JOTCSA. 2018; 5(3): 1071-1082.

RESEARCH ARTICLE 